ARTICLE

https://doi.org/10.1038/s41467-018-07705-w

\title{
Host-targeted niclosamide inhibits C. difficile virulence and prevents disease in mice without disrupting the gut microbiota
}

John Tam¹,2, Therwa Hamza ${ }^{3}$, Bing Ma ${ }^{4}$, Kevin Chen (1) ${ }^{3}$, Greg L. Beilhartz ${ }^{1}$, Jacques Ravel (1) ${ }^{4}$, Hanping Feng ${ }^{3}$ \& Roman A. Melnyk 1,2

Clostridium difficile is the leading cause of nosocomial diarrhea and colitis in the industrialized world. Disruption of the protective gut microbiota by antibiotics enables colonization by multidrug-resistant $C$. difficile, which secrete up to three different protein toxins that are responsible for the gastrointestinal sequelae. Oral agents that inhibit the damage induced by toxins, without altering the gut microbiota, are urgently needed to prevent primary disease and break the cycle of antibiotic-induced disease recurrence. Here, we show that the anthelmintic drug, niclosamide, inhibits the pathogenesis of all three toxins by targeting a host process required for entry into colonocytes by each toxin. In mice infected with an epidemic strain of $C$. difficile, expressing all three toxins, niclosamide reduced both primary disease and recurrence, without disrupting the diversity or composition of the gut microbiota. Given its excellent safety profile, niclosamide may address an important unmet need in preventing $C$. difficile primary and recurrent diseases.

\footnotetext{
${ }^{1}$ Molecular Medicine, Hospital for Sick Children, 686 Bay St., Toronto, ON M5G OA4, Canada. ${ }^{2}$ Department of Biochemistry, University of Toronto, Toronto, ON M5S 1A8, Canada. ${ }^{3}$ Department of Microbial Pathogenesis, University of Maryland Dental School, Baltimore, MD 21201, USA. ${ }^{4}$ Institute for Genome Sciences, University of Maryland School of Medicine, Baltimore, MD, USA. Correspondence and requests for materials should be addressed to R.A.M. (email: roman.melnyk@sickkids.ca)
} 
C lostridium difficile is a spore-forming Gram-positive bacterium that causes a range of gastrointestinal diseases, typically in individuals that have taken a course of broadspectrum antibiotics, which lowers the diversity of the protective resident GI microbiota ${ }^{1}$. In the resulting aftermath, opportunistic C. difficile colonizes the lower GI tract of susceptible individuals and secretes up to three gut-damaging toxins, including two large homologous toxins $\mathrm{TcdA}$ and $\mathrm{TcdB}$, and in the case of epidemic strains of $C$. difficile-such as ribotype 027 (RT027) ${ }^{2}$ and ribotype $078(\mathrm{RT} 078)^{3}-$ a third, unrelated binary toxin called $\mathrm{CDT}^{4}$. In recent years, $C$. difficile has become a major public health concern, due to the proliferation and global spread of epidemic strains, which are associated with increased morbidity and mortality ${ }^{5}$. The increased virulence of these strains has been attributed to several factors, including acquisition of mutations in $g y r B$ that result in resistance to fluoroquinolones ${ }^{5}$; increased expression of toxins ${ }^{6,7}$; production of a more cytotoxic form of $\mathrm{TcdB}^{8}$; and, expression of $\mathrm{CDT}^{2}$.

Extensive experimental and epidemiological evidence support a role for toxins as the primary determinants of disease pathogenesis. Isogenic knockout studies, in which toxins were deleted individually, or in combination, showed that TcdA and TcdB alone are sufficient to cause fulminant disease in hamsters 9,10 , whereas CDT appears to contribute to virulence in combination with $\mathrm{TcdA}$ or $\mathrm{TcdB}^{11}$. Importantly, knockout of all three toxins renders $C$. difficile completely avirulent.

The inextricable link between antibiotic-induced dysbiosis of the GI microbiota and infection by $C$. difficile, together with the well-validated role of toxins in driving disease pathogenesis, provide strong rationale and validation for targeting the actions of the $C$. difficile toxins as a novel approach to treat or prevent $C$. difficile infection (CDI). Despite early clinical setbacks with nonspecific polymers meant to sequester the toxins in the GI tract $^{12}$, the monoclonal antibody bezlotoxumab (ZinplavaMerck), which binds to and blocks TcdB following toxin-induced damage of the gut lining ${ }^{13}$, was recently approved for use in CDI patients for reducing recurrence ${ }^{14,15}$. This important clinical validation of toxin-targeting approaches for treating $C$. difficile recurrence has fueled efforts to develop next-generation antitoxins that are orally-bioavailable (i.e., small molecules), have a greater spectrum of activity against all $C$. difficile toxins, and potentially be used prior to, or during a suspected primary infection. Ideally, such a therapeutic, in addition to having an impeccable safety profile in humans, would not itself affect the composition of the protective gut microbiota, which is ultimately required to prevent further re-infection. Moreover, with the emergence of new $C$. difficile ribotypes, such as RT033, that do not express $\mathrm{TcdB}$, but are nevertheless pathogenic ${ }^{16-20}$, it would be desirable to have a single agent with the above characteristics that is also able to prevent TcdA- and/or CDT-induced pathogenesis.

In this study, we screened libraries of approved drugs in a phenotypic screen of TcdB-induced cell rounding with the goal of identifying small molecules that may potentially be repurposed for treating CDI through direct toxin inhibition. Recent drug repurposing phenotypic screens have led to discoveries of potential new candidate therapies for a number of infectious diseases, including for giardiasis ${ }^{21}$, Zika virus infection ${ }^{22}$, Ebola virus disease ${ }^{23}$, and Hepatitis $\mathrm{C}$ infection ${ }^{24}$. Here, among the panel of hits identified that completely protected cells from TcdB intoxication, we identified niclosamide, a widely used anthelmintic drug approved by the US FDA for treating intestinal infections of tapeworms ${ }^{25}$. Based on its excellent safety profile $\mathrm{e}^{26,27}$, and its preferential biodistribution in the colon resulting from its poor absorption in the GI tract ${ }^{26}$, we investigated niclosamide as an oral toxin-neutralizing treatment for CDI.

\section{Results}

High-throughput screen for inhibitors of TcdB intoxication. Intoxication by $\mathrm{TcdB}$ toxin is a multistep process, involving four functionally-distinct toxin domains, and several host-factors and processes (Fig. 1a). The intoxication of cells leads first to cytopathic effects (i.e., rounding of cells) ${ }^{28}$ within $1-3 \mathrm{~h}$, and later, cytotoxic effects (i.e., apoptosis) after $24 \mathrm{~h}^{29}$. To identify small molecules that protected cells from $\mathrm{TcdB}$, we employed a highthroughput assay of TcdB-induced cell rounding that we previously developed, which quantifies the extent of rounding of human lung fibroblasts treated with cytopathic doses of $\mathrm{TcdB}$ (i.e., $1 \mathrm{pM}$ for $3 \mathrm{~h}$ ) using high-content imaging analysis ${ }^{30}$. To increase the probability of identifying compounds with suitable properties for subsequent in vivo studies and beyond, we screened the Library of Pharmacologically Active Compounds (1280 compounds) and the Microsource Library Spectrum Collection (2360 compounds)-libraries consisting of approved drugs and pharmacologically active molecules with known targets and pharmacological properties.

From the 60 compounds that protected cells from TcdB by greater than the statistical cut-off of three standard deviations of the mean of the data (Supplementary Figure 1), we triaged drugs with undesirable mechanisms-of-action (i.e., antibiotics and antiseptics), and those that are known to be toxic or poorly tolerated in humans. Emerging from this prioritization were the three related salicylanilide anthelmintic drugs: niclosamide $(71 \%$ inhibition), closantel (60\% inhibition), and oxyclozanide (43\% inhibition) (Fig. 1b, c); drugs that act on parasites within the GI lumen, and that have well-documented safety margins in humans. Among these salicylanilides, niclosamide was the most potent inhibitor of TcdB-induced cell-rounding, protecting cells with an $\mathrm{EC}_{50}=0.51 \pm 0.03 \mu \mathrm{M}$ (values represent mean \pm s.e.m., $n=5$ ) (Fig. 1d). Protection from TcdB-induced cell-rounding by niclosamide was complete; human IMR-90 fibroblasts that were co-incubated with niclosamide and $\mathrm{TcdB}$ were indistinguishable from cells that had not received toxin (Fig. 1e). To evaluate the extent of protection by niclosamide against different amounts of TcdB (reflecting the range of toxin levels that might be experienced during an infection), cells were treated with a range of TcdB concentrations at different fixed doses of niclosamide. In the absence of drug, TcdB dose-dependently induces cell rounding with an $\mathrm{EC}_{50}=0.8 \mathrm{pM}$ (Fig. 1f). In the presence of increasing concentrations of niclosamide, the amount of TcdB required to reach equivalent levels of rounding increased dramatically. Remarkably, above the $\mathrm{EC}_{50}$ of niclosamide, cells were completely protected from TcdB by over three orders-ofmagnitude, corresponding to a protection factor $(\mathrm{PF})>5000$ (Fig. 1f).

Next, we tested the ability of niclosamide and the more watersoluble ethanolamine salt form of niclosamide, niclosamide ethanolamine $(\mathrm{NEN})^{26}$ to maintain the integrity of human epithelial colorectal cells (CaCo-2 cells) that were treated with TcdB. Treatment of a confluent monolayer of $\mathrm{CaCo}-2$ cells with $\mathrm{TcdB}$ results in disruption of monolayer integrity and loss of trans-epithelial resistance ${ }^{31}$ within hours of application as a result of GTD-induced actin depolymerization. Disruption of the monolayer integrity by TcdB was prevented by co-treatment with niclosamide (Supplementary Figure 2). Furthermore, NEN prevented the TcdB-induced disruption of Caco- 2 monolayers maintaining barrier function to untreated levels (Fig. 1g).

Mechanism of TcdB neutralization by niclosamide. To elucidate the mechanism by which niclosamide inhibits TcdB-induced cell rounding, we carried out a series of assays that evaluate each step of the intoxication pathway in isolation (Fig. 1a). Though 
a
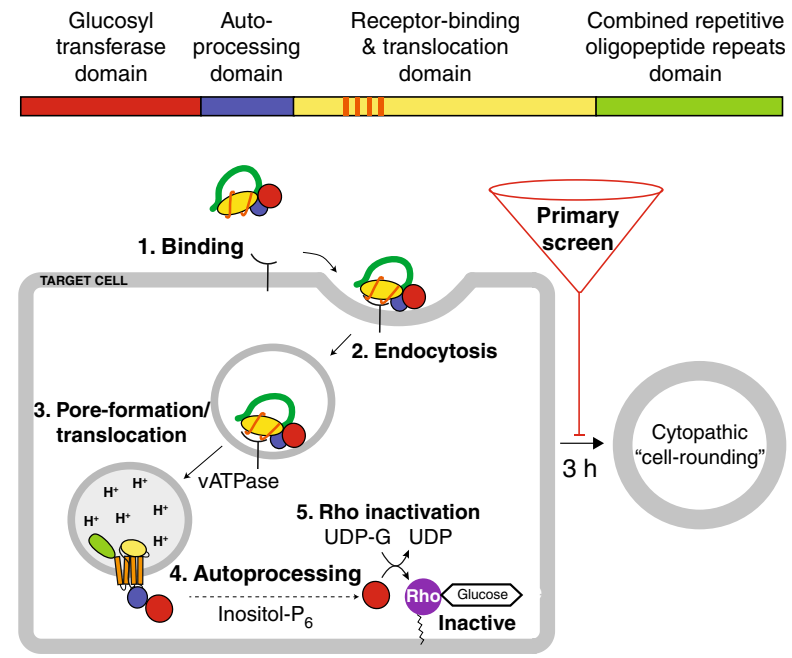

e

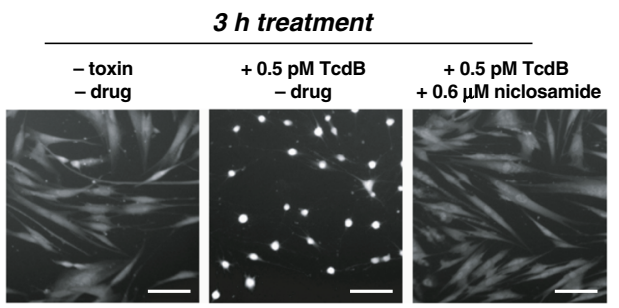

Human IMR-90 fibroblasts f

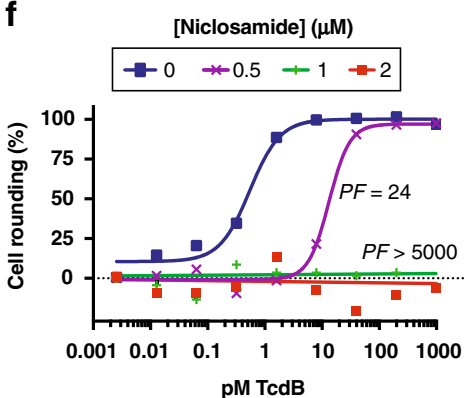

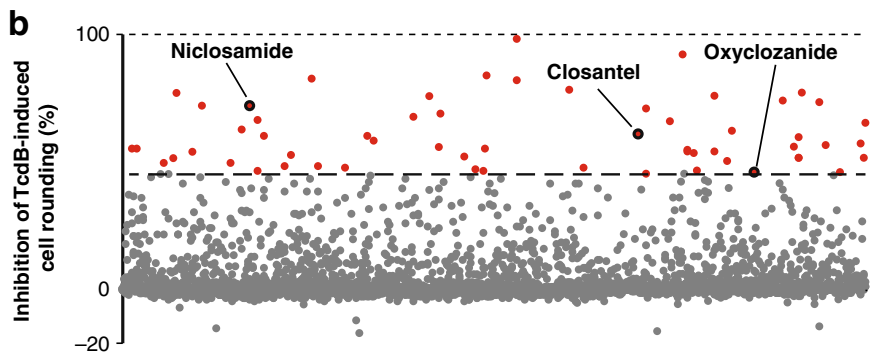

C
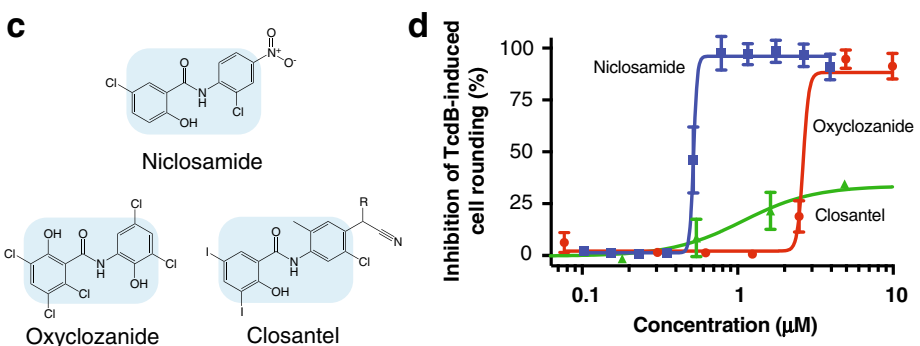

g

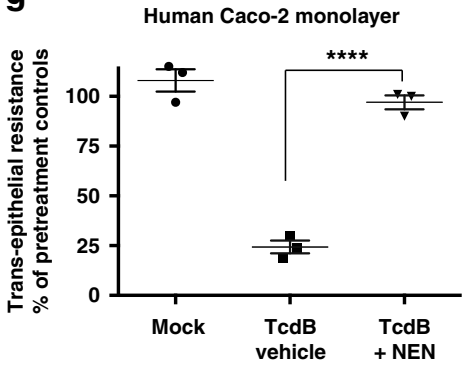

Fig. 1 Identification of niclosamide from a high-throughput screen of TcdB-induced cell-rounding. a Top, domain architecture of TcdB, highlighting the four domains involved in TcdB-induced cell-rounding. Membrane-insertion elements that are thought to form the translocation pore within endosomes ${ }^{33}$ are depicted as orange vertical lines within the receptor-binding and translocation domain. Bottom, steps involved in TcdB intoxication of human cells. Chemical libraries were screened at $40 \mu \mathrm{M}$ using an algorithm developed previously ${ }^{30}$. b Results from high-throughput screening of 3580 drugs from the LOPAC and Microsource collections. A statistical cutoff of $43 \%$ inhibition of cell rounding was based on identification of molecules that were greater than 3-standard deviations above the mean of the data. The salicylanilide anthelmintic drugs niclosamide, closantel, and oxyclozanide were among the 60 hits identified. c Chemical structures of niclosamide, oxyclozanide, and closantel. d Dose titration curves of the inhibition of cell-rounding by niclosamide, oxyclozanide, and closantel $(n=5 ; n=3$ for clostantel). Values represent mean \pm s.e.m. e Representative images of human IMR-90 fibroblasts from at least 8 experiments. Cells were pre-treated with DMSO or niclosamide 15 min before treatment with buffer or $0.5 \mathrm{pM}$ TcdB and images were collected $3 \mathrm{~h}$ later. White bars represent $100 \mu \mathrm{m}$. f Intoxication of human IMR-90 cells by TcdB in the presence of different doses of niclosamide after 3-h. Protection

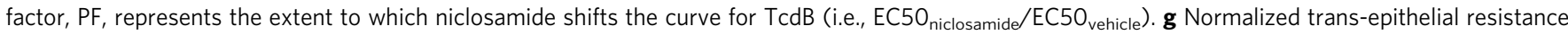
measurements in human $\mathrm{CaCo}-2$ cells, $6 \mathrm{~h}$ post-treatment. $5 \mu \mathrm{M}$ NEN preserved significantly increased resistance across Caco- 2 monolayer cells compared to mock control values $(n=3)$. Values represent mean \pm s.e.m. ${ }^{\star \star \star \star} P<0.0001$ (unpaired $t$-test with Welch's correction)

niclosamide dose-dependently inhibits Racl glucosylation in cells (Fig. 2a), no direct inhibition of GTD enzymatic activity is observed in vitro up to $10 \mu \mathrm{M}$ niclosamide, indicating that inhibition occurs at a step upstream of Racl glucosylation (Fig. 2b). The release of GTD by the APD domain, the step immediately preceding Racl glucosylation, was also unaffected by up to $10 \mu \mathrm{M}$ niclosamide in an in vitro assay of inositol hexakisphosphateinduced autoprocessing (Fig. 2c).

Consistent with niclosamide not acting through inhibition of either GTD or APD activity, we found that niclosamide inhibited TcdB-induced necrosis (Fig. 2d) - a cellular phenotype that is independent of GTD and APD activity, but dependent on cellsurface binding, uptake into endosomes, and $\mathrm{pH}$-dependent poreformation in endosomes for full activity ${ }^{32,33}$. Using mammalian cells expressing all three known receptors, we found that niclosamide did not affect $\mathrm{TcdB}$ binding to the cell surface (Fig. 2e). Moreover, we saw no evidence for a direct binding interaction between niclosamide and $\mathrm{TcdB}$ (Supplementary
Figure 3). Taken together, these data point to niclosamide acting on the host, and through inhibition of the pore-formation process. Niclosamide has been reported to mildly increase the $\mathrm{pH}$ of endosomes through a unique "proton-shuttle" mechanism ${ }^{34}$, which is distinct from other modes of endosomal deacidification, such as lysosomotropism. Using fluorescent LysoTracker dye, a lysosomotropic molecule that accumulates in acidic vesicles, we found that pre-treating cells with either niclosamide or NEN indeed reduces LysoTracker fluorescent staining, consistent with these molecules increasing the $\mathrm{pH}$ of endosomal compartments (Fig. 2f). Importantly, the dose-dependent decrease in endosomal $\mathrm{pH}$ by niclosamide and NEN overlapped with the dose-titration curves for inhibition of cell rounding and necrosis (Fig. $2 \mathrm{~g}$ ).

Niclosamide protects cells from all three $C$. difficile toxins. The determination that niclosamide inhibited TcdB at the level of the host endosome prompted us to consider the intriguing possibility 
a

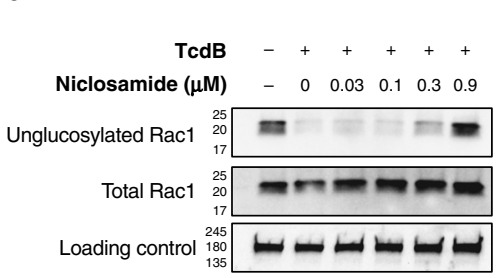

b

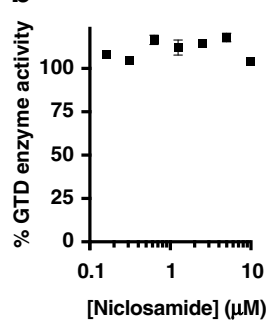

c

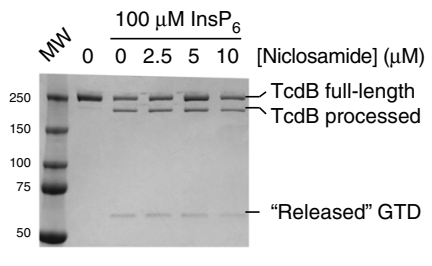

d

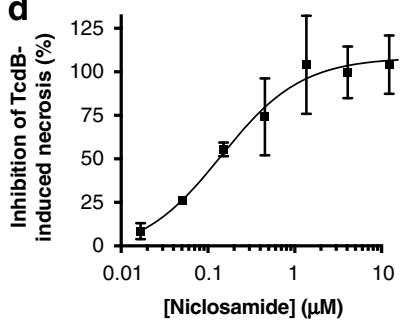

e

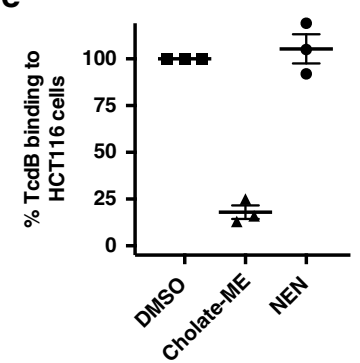

f

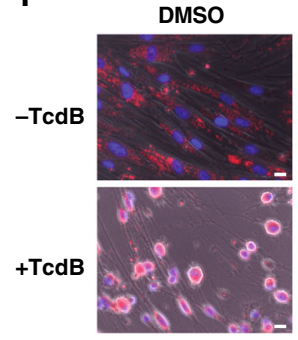

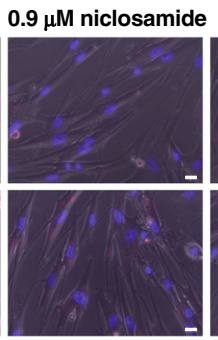

$0.9 \mu \mathrm{M}$ NEN

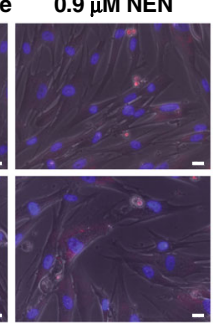

g

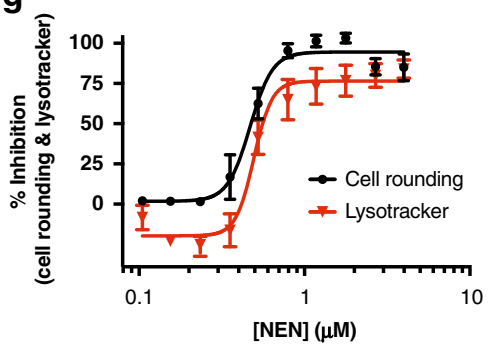

Fig. 2 Mechanism-of-action of niclosamide inhibition of TcdB intoxication. a Western blot image for intracellular Rac1 glucosylation ( $n=3$ ). IMR-90 cells were treated with niclosamide (doses indicated) for $15 \mathrm{~min}$, followed by treatment with $0.5 \mathrm{pM}$ TcdB. Cells were harvested as described in Methods. Mab102, which recognizes un-glucosylated Rac1 in cells, shows a dose-dependent re-appearance with increasing concentration of niclosamide, relative to total Rac1 levels $(n=2)$. b In vitro GTD glucosyltransferase assay. Recombinant GTD was incubated for 30 min with different doses of niclosamide ( $n=3$ ). Activity was measured as described in Methods. Niclosamide at concentrations up to $10 \mu \mathrm{M}$ did not directly affect GTD activity itself. c In vitro autoprocessing assay. Compound or DMSO was added to $100 \mathrm{nM} \mathrm{TcdB}$, and pre-incubated for $60 \mathrm{~min}$. To initiate auto-processing, InsP6 was added (100 $\mu \mathrm{M}$ ) and incubated @ $37^{\circ} \mathrm{C}$ for 20 min before stopping with Laemlii sample buffer. Niclosamide at concentrations up to $10 \mu \mathrm{M}$ did not affect auto-processing $(n=2)$. d Niclosamide was titrated in IMR90 cells in the presence of $1 \mathrm{nM}$ TcdB for 3-h. Loss of cellular ATP as a marker of high dose toxicity was measured by adding CellTiter-Glo reagent (Promega). Niclosamide inhibited TcdB-induced necrosis with an $\mathrm{IC}_{50}=0.15 \mu \mathrm{M}$. Bars represent $\mathrm{SEM}$ of two experiments. e Cell surface binding assay was carried out as described in Methods. Methylcholate, but not NEN, prevented surface binding of TcdB to cells $(n=3)$. $\mathbf{f}$ LysoTracker assay was carried out as described in Methods on IMR90 cells in the absence (top panels) or presence (bottom panels) of $1 \mathrm{pM}$ TcdB. Acidic compartments are stained in red, while the nuclei are stained in blue. Cells treated with TcdB and vehicle are rounded and raised in comparison to untreated cells. Cells treated with niclosamide or NEN did not exhibit LysoTracker staining, and were protected from TcdB-induced cell rounding. White bars represent $20 \mu \mathrm{m}$. $\mathbf{g}$ Titration of NEN by LysoTracker Red DND-99. The LysoTracker fluorescence at ex/em $574 / 594$ was measured on a Spectramax i3x plate reader (Molecular Devices). The $\mathrm{IC}_{50}$ s by cell rounding and LysoTracker assays were 0.47 and $0.49 \mu \mathrm{M}$, respectively. Bars represent SEM of four experiments

Table 1 Inhibition of $C$. difficile toxin-induced cytotoxicity by niclosamide and NEN

\begin{tabular}{|c|c|c|c|c|c|}
\hline & TcdB $_{012}$ & $\mathrm{TcdB}_{078}$ & $\mathrm{TcdB}_{027}$ & TcdA & CDT \\
\hline Niclosamide $I_{50}(\mu \mathrm{M})$ & $0.44 \pm 0.05$ & $0.5 \pm 0.2$ & $0.4 \pm 0.1$ & $0.5 \pm 0.1$ & $0.53 \pm 0.08$ \\
\hline NEN IC ${ }_{50}(\mu \mathrm{M})$ & $0.43 \pm 0.03$ & $0.45 \pm 0.09$ & $0.5 \pm 0.1$ & $0.55 \pm 0.08$ & $0.50 \pm 0.09$ \\
\hline
\end{tabular}

that niclosamide might additionally block the actions of TcdA and CDT, both of which require endosomal acidification for pore-formation and intracellular entry. Indeed, niclosamide and NEN completely protected cells from TcdA-induced cell rounding, and from CDT-induced damage (i.e., depolymerization of the actin cytoskeleton), at the same doses that protect cells from TcdB (Table 1 and Supplementary Figure 4). NEN also protected cells against a form of TcdB derived from hypervirulent strains of $C$. difficile, which have been shown to enter cells at an earlier stage in endocytosis $^{8}$ (Table 1).

Demonstrating protection against CDT, a toxin that bears no structural or functional similarities to TcdA and TcdB, other than requiring low $\mathrm{pH}$ to escape endosomes, further supports the mechanism-of-action for niclosamide. More importantly, this finding suggests that niclosamide, as a single entity, could potentially protect from infection and disease by all pathogenic $C$. difficile strains, expressing any combination of toxins, in vivo.
NEN reduces disease by an epidemic strain of $C$. difficile in mice. Mice preconditioned with antibiotics and challenged with C. difficile develop typical CDI (weight loss, diarrhea, death) in the absence of any therapeutic countermeasures ${ }^{1}$. To test the hypothesis that niclosamide is capable of preventing disease induced by strains expressing multiple toxins, we evaluated the efficacy of NEN in protecting against CDI in a murine model challenged with the hypervirulent strain UK1 (RT027), which expresses TcdA, TcdB, and CDT. Infected mice were treated with either water (control) or NEN (at different doses; 2, 10, and $50 \mathrm{mg}$ per $\mathrm{kg}$ ) via oral gavage $4 \mathrm{~h}$ post spore challenge and for 3 consecutive days after spore challenge (Fig. 3a). Typical symptoms of CDI in murine models include severe weight loss on days 2 and 3 post-challenge accompanied with diarrhea and high mortality rate in sham groups. All doses of NEN tested significantly protected mice from weight loss compared to control group (Fig. 3b). NEN protected mice from death in a dose-dependent manner, 
a

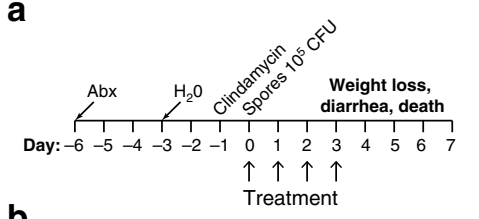

b

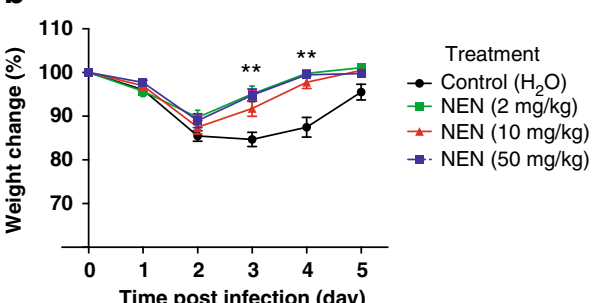

C

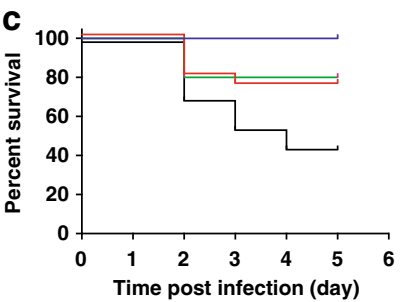

d

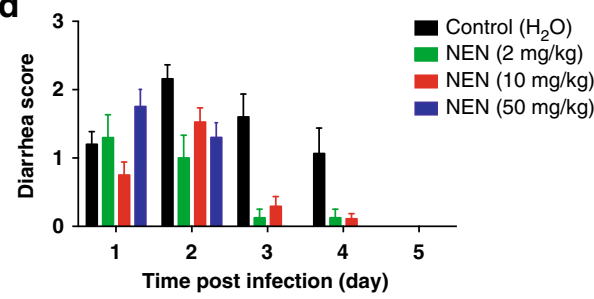

- Control $\left(\mathrm{H}_{2} \mathrm{O}\right)$

$-\mathrm{NEN}(2 \mathrm{mg} / \mathrm{kg})$
$-\mathrm{NEN}(10 \mathrm{mg} / \mathrm{kg})$ e

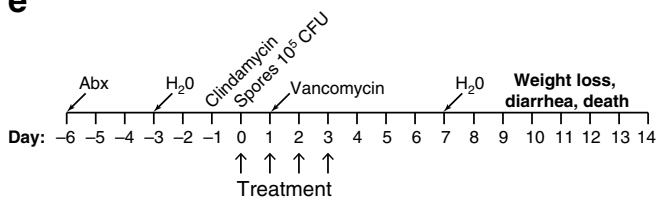

f

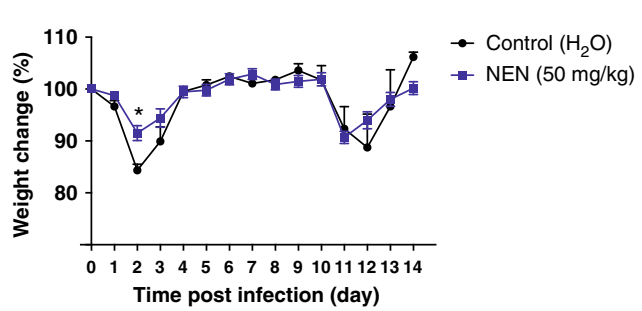

- NEN (50 mg/kg)

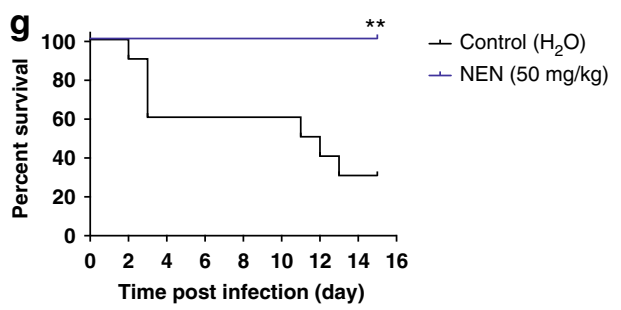

h

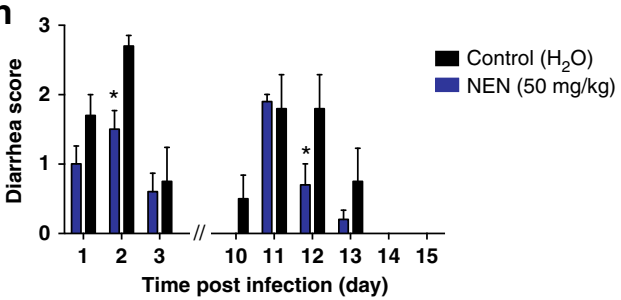

Fig. 3 Niclosamide ethanolamine (NEN) is protective in primary and recurrent CDI. a Protocol schematic for primary CDI model. b Weights of mice after challenge with $\mathrm{C}$. difficile spores $\left(10^{5} \mathrm{CFU} / \mathrm{mL}\right.$ ) on day 0 . Mice were treated with vehicle (5\% DMSO) or NEN (in 5\% DMSO) at different doses ( 2,10 , and $50 \mathrm{mg}$ per $\mathrm{kg}$ ). Each point is the mean SE from day 0. c Mouse survival as determined by a log-rank/Mantel-Cox test. d Diarrhea score of infected mice 5 . e Protocol schematic for recurrent CDI model. $\mathbf{f}$ Weights of mice after $C$. difficile spore challenge (day 0 ) followed with vancomycin in their drinking water for recurrence CDI model. $\mathbf{g}$ Survival of infected mice treated and un-treated with NEN (50 mg per $\mathrm{kg}$ ). $\mathbf{h}$ Diarrhea score of infected mice. ${ }^{\star} p<0.05$;

${ }^{\star *} p<0.01$ using unpaired Student $t$-test. Data represent 10 mice/group

\section{Table 2 MIC values for niclosamide, NEN, and vancomycin} on Clostridial species

\begin{tabular}{lll} 
Organism (strain) & Vancomycin $(\boldsymbol{\mu g} / \mathbf{m L})$ & NEN $^{\mathbf{a}}(\boldsymbol{\mu g} / \mathbf{m L})$ \\
\hline C. difficile R20291 (027) & 1 & $>19$ \\
C. difficile M68 (017) & 2 & $>19$ \\
C. clostridioforme ATCC25537 & 4 & $>19$ \\
C. sporogenes ATCC3584 & 8 & $>19$ \\
\hline Data are $n=3$ & & \\
a19 $\mu \mathrm{g} / \mathrm{mL}=50 \mu \mathrm{M}$ & & \\
\hline
\end{tabular}

with all mice in the $50 \mathrm{mg}$ per $\mathrm{kg}$ group remarkably surviving infection, compared to only $45 \%$ for control group (Fig. 3c). These results closely tracked the wet tail and diarrhea scores, which were significantly lower in NEN-treated groups (Fig. 3d).

Symptomatic recurrence of CDI, which occurs in approximately one-in-four individuals, is a characteristic feature of CDI that complicates eradication and management of $C$. difficile ${ }^{2}$. We assessed whether NEN (50 $\mathrm{mg}$ per $\mathrm{kg}$ ) could prevent recurrence in a mouse model of recurrent CDI in which infected mice are treated with vancomycin $(0.5 \mathrm{mg}$ per $\mathrm{mL})$ in their drinking water starting on day 1 ongoing for 6 days after spore challenge (Fig. 3e). NEN treatment was given via oral gavage $4 \mathrm{~h}$ post spore challenge and for 3 consecutive days after spore challenge. Both groups (NEN-treated and un-treated) started losing weight on day 4 after receiving vancomycin water (i.e., day 11 post spore challenge) (Fig. 3f). As above, all mice in the NEN group survived from $C$. difficile challenge, whereas more than $60 \%$ of mice in the control group became moribund (Fig. 3g, h). After resolution of symptoms, both groups began to lose weight at day 11; however, NEN-treated mice displayed less severe diarrhea scores, and importantly all NEN-treated mice survived recurrence.

NEN does not affect $C$. difficile growth in vitro. As salicylanilide derivatives have been reported previously to have antimicrobial activity against certain Gram-positive bacteria ${ }^{35,36}$, we next carried out a series of experiments to address, whether NEN, specifically, had any antibacterial activity against $C$. difficile that may have contributed to the protective effects seen in vivo. To this end, we measured the minimum inhibitory concentrations (MICs) of NEN on individual strains of Clostridium species using the gold-standard anaerobic agar dilution assay ${ }^{37}$. No antimicrobial activity was seen for NEN up to $19 \mu \mathrm{g} / \mathrm{mL}$ (i.e., $50 \mu \mathrm{M}$ NEN) against either of the two strains of $C$. difficile tested (017 and 027), or against the two non-pathogenic Clostridium species tested (Table 2). These results were confirmed against a larger panel of $C$. difficile strains, where we saw no activity for NEN up to $32 \mu \mathrm{g} / \mathrm{mL}$ (i.e., $84 \mu \mathrm{M})$ (Table 3). While these data indicate that 


\begin{tabular}{|c|c|c|c|c|}
\hline \multirow{2}{*}{$\begin{array}{l}\text { Organism ATCC } \\
(M M X \text { No. })^{a}\end{array}$} & \multicolumn{4}{|c|}{$M I C$ in $\mu \mathrm{g} / \mathrm{mL}$} \\
\hline & Fidaxomicin & Vancomycin & Metronidazole & NEN \\
\hline $\begin{array}{l}\text { C. difficile } 70005 \\
\text { (4381) }\end{array}$ & $0.06^{b}$ & $4^{c}$ & $0.5^{d}$ & $>32$ \\
\hline C. difficile (8261) & 0.06 & 2 & 0.5 & $>32$ \\
\hline C. difficile (8262) & 0.12 & 4 & 0.25 & $>32$ \\
\hline C. difficile (8263) & 0.06 & 2 & 0.25 & $>32$ \\
\hline C. difficile (8341) & 0.06 & 1 & 0.25 & $>32$ \\
\hline C. difficile (8336) & 0.12 & 4 & 0.5 & $>32$ \\
\hline C. difficile (8337) & 0.06 & 1 & 0.25 & $>32$ \\
\hline C. difficile (8338) & 0.12 & 4 & 2 & $>32$ \\
\hline C. difficile (8339) & 0.12 & 4 & 4 & $>32$ \\
\hline C. difficile (8340) & 0.12 & 2 & 0.5 & $>32$ \\
\hline \multicolumn{5}{|c|}{$\begin{array}{l}\text { a Micromyx Isolate Number } \\
\text { bCLSI QC range }(0.06-0.25) \\
\text { cCLSI QC range }(0.5-4) \\
\text { dCLSI QC range }(0.125-0.5)\end{array}$} \\
\hline
\end{tabular}

NEN does not affect $C$. difficile growth directly, an important feature to demonstrate for NEN, or any would-be C. difficile therapeutic, is the lack of effect on the gut microbiota.

NEN effects on the gut microbiota in vivo. To directly address whether NEN had any effects on the gut microbiota that may contribute to disease pathogenesis, we next evaluated the effect of NEN treatment on the composition and structure of the gut microbiota in mice under various situations. First, in mice that were not infected with $C$. difficile, we investigated the effects of NEN at the highest dose tested in the efficacy study (i.e., $50 \mathrm{mg}$ per $\mathrm{kg}$ ), and compared this with both vancomycin and vehicle control. As shown in Fig. 4a, gut microbiota diversity in NENtreated and control mice are only slightly but statistically significant different on day 3 , however, the difference is minimal compared to the large reduction observed after vancomycin treatment. This small difference disappears on day 6 , while that observed in the vancomycin-treated group remains high. Further, ordination analysis shows the minimal effect of NEN and the large effect of vancomycin on the gut microbiota structure at all time points post-treatment compared to controls (Supplementary Figure 5a). Vancomycin treatment dramatically lowered the diversity of the microbiota, shifting the composition to high relative abundance of Lactobacillaceae and Enterobacteriaceae (Fig. 4a) as seen previously ${ }^{38}$. Thus, we conclude that NEN treatment has a minimal effect on the gut microbiota.

Next, we evaluated the effects of NEN on the gut microbiota in C. difficile-infected mice that had been pre-treated with an antibiotic cocktail 6, 5, and 4 days prior to infection and clindamycin the day prior to infection (Fig. $4 \mathrm{~b}-\mathrm{d}$ ). The diversity of the microbiota following NEN treatment $(50 \mathrm{mg}$ per $\mathrm{kg}$ ) on days 1,2 , and 3 post-infection was indistinguishable from the water control group (Fig. 4b). Further, the composition and structure of the gut microbiota on day 6 post-infection did not differ from water treatment (Fig. $4 \mathrm{~b}$ and Supplementary Figure 5), comprising of high relative abundance of Lactobacillaceae, Bifidobacteriaceae, Clostridiales, and Bacteroidales, and decreased relative abundance of Enterobacteriaceae (mainly Escherichia coli) (Supplementary Figure 5 and Supplementary Figure 6). Linear discriminant analysis (LDA) effect size (LEfSe) analysis ${ }^{39}$ only identified members of the genus Bacteriodes out of 154 phylotypes as significantly more abundant on day 6 in water control than in NEN treated mice (Supplementary Figure 7).
Having shown that NEN does not affect the structure or composition of the microbiota, we next asked whether NEN (alone or combination with vancomycin) was potentially able to help restore the gut microbiota during the resolution phase of infection. To this end, we compared the effects of NEN $(50 \mathrm{mg}$ per $\mathrm{kg})+$ vancomycin $(0.5 \mathrm{mg}$ per $\mathrm{kg})$, and NEN ( $50 \mathrm{mg}$ per $\mathrm{kg}$ ) alone, to vancomycin alone, in the recurrent $C$. difficile model. As expected, the diversity of the gut microbiota after vancomycin treatment alone remained low throughout the treatment cycle and was dominated by Lactobacillaceae (Fig. 4c, d). Whereas the addition of NEN ( $50 \mathrm{mg}$ per $\mathrm{kg}$ ) to vancomycin $(0.5 \mathrm{mg}$ per $\mathrm{kg}$ ) showed no benefits to the microbiota compared to vancomycin alone (Fig. 4c), treatment with NEN (50 mg per kg) alone resulted in a significant increase in diversity post-treatment and during resolution of infection (Fig. 4d), indicating that NEN may have additional benefits on the gut microbiota as a stand-alone therapy.

\section{Discussion}

The global spread of epidemic strains of $C$. difficile capable of causing outbreaks and life-threatening infections is a recent phenomenon that has been brought on, in part, by modern human practices. The widespread introduction of the food additive trehalose, shortly before the emergence of epidemic RT027 and RT078, has been proposed to have contributed to selecting for these strains and increasing their virulence ${ }^{7}$. Similarly, the use and misuse of antibiotics have further accelerated the enrichment of multidrug-resistant variants of $C$. difficile, whilst disrupting the protective microbiota that normally prevents such infections ${ }^{40}$. As a result, $C$. difficile continues to increasingly become more widespread, more virulent, and more difficult to treat with traditional eradication approaches (i.e., antibiotics). The notion of targeting the virulence determinants of C. difficile has emerged as an attractive alternative strategy to treat $\mathrm{CDI}^{30,41-43}$, especially given the role that toxins play in all aspects of disease pathogenesis ${ }^{9-11,44}$. The recent clinical demonstration of disease recurrence attenuation by the injectable TcdB-targeted antibody bezlotoxumab ${ }^{15}$ supports these approaches and has fueled efforts to identify next generation antivirulence therapeutics. In particular, more convenient oral agents (i.e., small molecules) that can be dosed at all stages of disease are highly sought after. Moreover, although targeting TcdB appears to be capable of decreasing recurrence, blocking the actions of TcdA and binary toxin, both of which contribute to disease pathogenesis in hypervirulent strains and appear to be sufficient for causing disease in strains lacking $\mathrm{TcdB}$ in certain cases, would be a highly desirable feature of any comprehensive would-be antivirulence strategy.

In this study, we performed a high-throughput screen of libraries containing FDA- and EMEA-approved drugs to identify small-molecules that protected cells from TcdB intoxication that could potentially be repositioned as orally-bioavailable therapeutics for treating CDI. Among the dozens of hits identified in the primary screen (Supplementary Figure 1), we noted that several were approved anti-parasitic drugs. From the most potent inhibitors of cell-rounding in this class, niclosamide was selected for further characterization based on its impeccable safety profile in humans, and known preferential distribution in the lower GI after oral dosing ${ }^{26}$, which we anticipated would be beneficial for targeting the gut-damaging toxins of $C$. difficile. Niclosamide is a remarkably well-studied molecule that has been shown to have a number of other biological activities in vitro that have prompted other investigations into translation into diseases including cancer $^{45-49}$, diabetes ${ }^{50}$ as well as other infectious diseases ${ }^{22}$. In most cases, however, the low systemic exposure of niclosamide, 
a

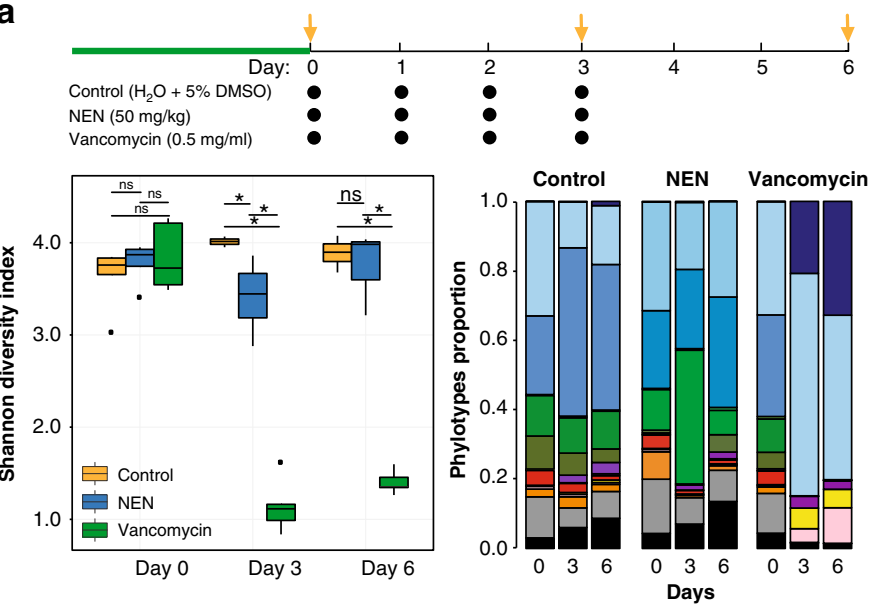

C

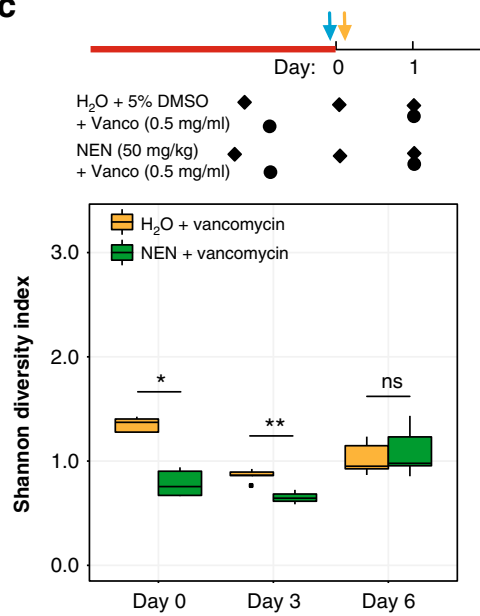

b
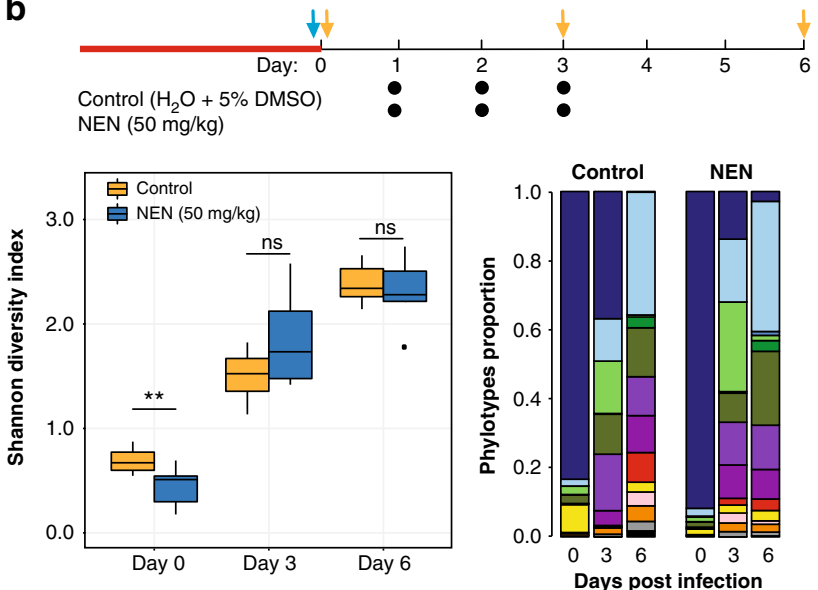

d
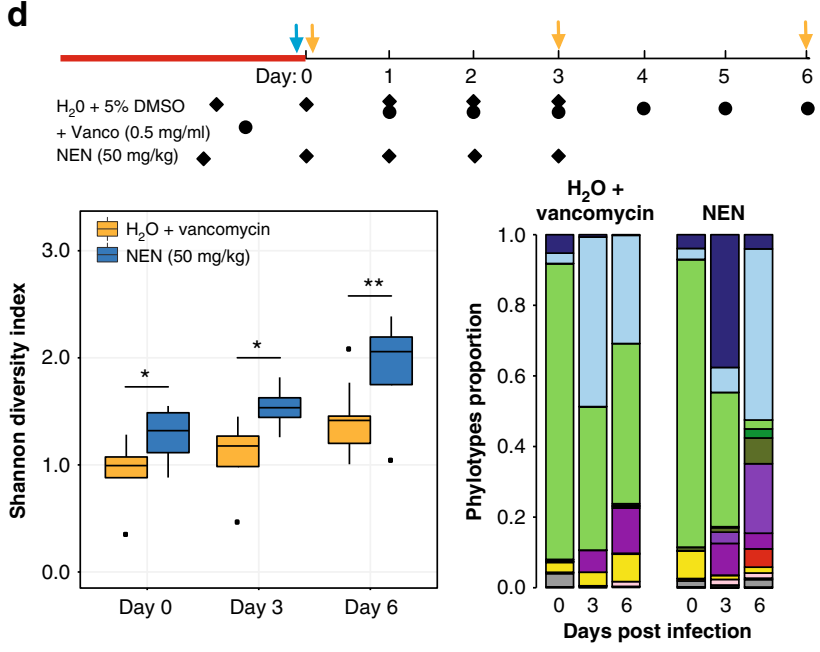

\begin{tabular}{|llllll}
\hline$\square$ Enterobacteriaceae & $\square$ Enterococcaceae & $\square$ Bacteroidaceae & $\square$ Alcaligenaceae & $\square$ Unclassified clostridiales & \\
$\square$ Lactobacillaceae & $\square$ Coriobacteraceae & $\square$ Porphyromonadaceae & $\square$ Verrucomicrobiaceae & $\square$ Others & $\downarrow$ Sampling day \\
$\square$ Bacteroidales S24-7 & $\square$ Bifidobacteriaceae & $\square$ Lachnospiraceae & $\square$ Erysipelotrichaceae & & $\downarrow$ c. difficile infection
\end{tabular}

Fig. 4 Effect of treatment on the gut microbiota diversity and composition. Experimental design is shown on top panel (red line indicates antibiotic cocktail treatment 6 days prior to the $C$. difficile infection for 3 days, followed by 2 days of water and clindamycin ( $30 \mathrm{mg}$ per $\mathrm{kg}$ ) the day prior to $\mathrm{C}$. difficile infection, green line indicates no treatment). Within-sample diversity was estimated using Shannon diversity index (ns denotes not significant, ${ }^{*} p$ value greater than 0.01 but not greater than $0.05,{ }^{\star \star} p$ value is less than 0.01 , unpaired Student $t$-test) The center line, bounds of box, and whiskers of the boxplot represents median, interquartile, and range of within-sample diversity. Each treatment group is the result of two experimental cages. Each barplot indicates the mean relative abundance of bacterial families with relative abundance $>1 \%$ from mice in two experimental cages. The Peptostreptococcaceae family of bacteria includes C. difficile and its abundance was consistently below $1 \%$ in all experiments and thus is included in others. a Control, NEN, and vancomycin treatment in the absence of $C$. difficile infection; b control (water with 5\% DSMO) and NEN treatment (50 mg per kg); c water with vancomycin and NEN treatment ( $50 \mathrm{mg}$ per $\mathrm{kg}$ ); d water with vancomycin and NEN treatment (50 mg per $\mathrm{mL}$ ) with vancomycin $(0.5 \mathrm{mg}$ per $\mathrm{mL}$ ). First sampling day (day 0 ) is $4 \mathrm{~h}$ after $\mathrm{C}$. difficile infection

which is likely a major contributor to its overall safety, has hampered its use for indications outside of the GI tract. Nevertheless, efforts have been undertaken to improve the bioavailability of niclosamide, using different salt forms, chemical modifications, and the use of nanoparticles ${ }^{51-53}$.

Here, we found that niclosamide provided protection to a variety of human cells from both TcdB-induced cell-rounding, and TcdB-induced necrosis. Moreover, due to its mechanism of inhibition of $\mathrm{TcdB}$ (i.e., targeting host endosomal $\mathrm{pH}$ through a proton-shuttle mechanism), niclosamide had the added major benefit of being equally effective against TcdA and CDT—both of which also require low $\mathrm{pH}$ for entry into the host. These features provided the unique opportunity to test niclosamide as a standalone agent against epidemic strains of $C$. difficile that are triple- positive for TcdA, TcdB, and CDT. In mouse models of CDI, we showed that NEN dose-dependently improved disease symptoms associated with both primary infection and recurrence, with full protection seen at $50 \mathrm{mg}$ per $\mathrm{kg} \mathrm{NEN}$. Of note, the reported median oral lethal dose $\left(\mathrm{LD}_{50}\right)$ for NEN in rats is $10,000 \mathrm{mg}$ per $\mathrm{kg}$ body weight ${ }^{27}$, further emphasizing the large therapeutic index for NEN in treating CDI. Finally, an important and significant finding in this study was that NEN had no major deleterious effects on the structure and composition of the microbiota (Fig. 4). Increased diversity of the gut microbiota was observed after NEN treatment, a feature that was thought to be unique to fecal microbiota transplantation and key to its success. Recovery of a diverse microbiota might in part contribute to its curative properties. Beyond this study and this indication, the findings 
presented here pave the way to future studies aimed at investigating the effects of NEN on other toxin-driven enteric diseases. Also, given the increasingly appreciated role that helminths play in interacting with and modulating the microbiome ${ }^{54,55}$, the results here highlight the importance of future studies aimed at understanding the effects of NEN on the microbiota during a helminth infection.

\section{Methods}

Cell lines, consumables, and reagents. Plasticware used for cell culture and enzyme assays were purchased from Corning. Streptavidin Hi-bind plates, Superblock buffer, SuperSignal West, and Quantablu peroxidase substrate were purchased from Thermo Pierce (Rockford, IL). Cell lines CHO-K1 (ATCC, Cat \#CCL-61), Vero (ATCC, Cat \#CCL-81), CaCo-2 (ATCC, Cat \#HTB-37), HCT-116 (ATCC, Cat \#CCL-247), and IMR90 (ATCC, Cat \#CCL-186) were purchased from ATCC (Manassas, VA). Anti-TcdB antibody (used at 1:2000 dilution) was purchased from R\&D Systems (Cat \#AF6246). Anti-Rac1 antibody Mab102 (used at 1:1000 dilution) was purchased from BD Biosciences (Mississauga, ON), Cat $\# 610651$. Anti-Rac1 antibody 23A8 (used at 1:1000 dilution) was purchased from Millipore (Cat \#05-389). Anti-ERK and Anti-EEA1 (used at 1:2000 dilution) were purchased from Cell Signalling Technologies (Cat \#137F5) and Millipore (Cat \#07182-25UL), respectively. Anti-mouse conjugated peroxidase antibody (used at 1:10,000 dilution) was purchased from GE Healthcare (Cat \#NXA931V). Antirabbit HRP (used at 1:10,000 dilution) was purchased from GE Healthcare (Cat \#NA934V). Anti-sheep HRP (used at 1:100,000 dilution) was purchased from Invitrogen (Cat \#81-8620). The Spectrum library, consisting of 2320 individual compounds formatted as $10 \mathrm{mM}$ solutions in DMSO, was purchased from Microsource (Gaylordsville, CT). The LOPAC ${ }^{1280}$ was purchased from SigmaAldrich (Oakville, ON)

Protein expression and purification. Expression and isolation of recombinant toxins were as described by Yang et al. ${ }^{56}$. Briefly, transformed Bacillus megaterium was inoculated into LB containing tetracycline and grown to an A600 of 0.7, followed by overnight xylose induction at $37^{\circ} \mathrm{C}$. Bacterial pellets were collected, resuspended with $20 \mathrm{mM}$ Tris $\mathrm{pH} 8 / 0.5 \mathrm{M} \mathrm{NaCl}$, and passed twice through an EmulsiFlex C3 microfluidizer (Avestin, Ottawa, ON) at 15,000 psi, then clarified by centrifugation at $18,000 \mathrm{~g}$ for $20 \mathrm{~min}$. TcdB was purified by nickel affinity chromatography followed by anion exchange chromatography using HisTrap FF and HiTrap Q columns (GE Healthcare, Baie D'Urfe, QC), respectively. Fractions containing TcdB were verified by SDS-PAGE, then pooled and diafiltered with a 100,000 MWCO ultrafiltration device (Corning) into $20 \mathrm{mM}$ Tris pH 7.5/150 mM $\mathrm{NaCl}$. Finally, glycerol was added to $15 \% \mathrm{v} / \mathrm{v}$, the protein concentration was estimated by A280 (using coefficient 288160), divided into single-use aliquots, and stored at $-80^{\circ} \mathrm{C}$.

For TcdA, cell lysates were prepared as described for full-length TcdB, and purification of the protein was by nickel affinity chromatography using HisTrap FF columns.

The pGEX-Rac1 plasmid (Addgene plasmid 12200) for expression of GST-Rac1 protein was previously described by Bagrodia et al. ${ }^{57}$ and obtained from Addgene (Cambridge, MA). The plasmid was transformed into E. coli BL21 DE3, and recombinant protein expression was achieved by induction of the culture with $0.1 \mathrm{mM}$ IPTG for $5 \mathrm{~h}$ at $30^{\circ} \mathrm{C}$. The cell pellet was recovered by centrifugation, resuspended with $5 \mathrm{~mL} / \mathrm{g}$ of pellet in $20 \mathrm{mM}$ Tris $\mathrm{pH} 7.5 / 150 \mathrm{mM} \mathrm{NaCl}$, and sonicated. The cell lysate was clarified by centrifugation, and the GST fusion protein was purified by chromatography through a GSTrap Fast Flow column (GE Healthcare, Baie D'Urfe, QC). Following elution with $10 \mathrm{mM}$ glutathione, fractions containing purified protein were pooled and stored at $-80^{\circ} \mathrm{C}$ in the presence of $15 \% \mathrm{v} / \mathrm{v}$ glycerol.

Arrayscan high content phenotypic screen. IMR90 cells were grown in EMEM (Wisent) supplemented with $10 \%$ FBS and penicillin-streptomycin (complete EMEM) and were seeded in 96-well Cellbind plates (Corning) at a density of 8000-10,000 cells/well. The next day, the media was exchanged with serum-free EMEM (SFM) containing $1 \mu \mathrm{M}$ CellTracker Orange CMRA (Molecular Probes). After $60 \mathrm{~min}$, excess dye was removed by media exchange with SFM. An Agilent Bravo liquid handler was used to deliver $0.4 \mu \mathrm{L}$ of compound from the Microsource library plate to the cell plate, immediately followed by $10 \mu \mathrm{L}$ of $100 \mathrm{pM}$ TcdB (diluted in SFM) to a final volume of $100 \mu \mathrm{L}$, representing a concentration of toxin previously established as $\sim$ EC99 levels of cytopathology. The cell plates were returned to the incubator for $3.5 \mathrm{~h}$ before imaging. CellTracker-labeled cells were evaluated on a Cellomics ArrayScan VTI HCS reader (Thermo Scientific, Waltham, MA) using the Target Acquisition mode, a 10 objective and a sample rate of 100 objects per well. After recording all image data, the cell rounding and shrinking effects of TcdB intoxication were calculated using the cell rounding index (CRI), a combined measure of the length to width ratio (LWR) and area parameters. The \% inhibition was calculated as the ratio between the sample well and the average toxin-untreated controls after subtracting the average DMSO control values. The $Z^{\prime}$ value was calculated using the equation $Z^{\prime}=1-\left[\left(3 s_{f}+3 s_{b}\right) /\left(\mu_{b}-\mu_{f}\right)\right]$, where $s=$ standard deviation, $\mu=$ average, $f=$ DMSO control, and $b=$ toxin-untreated control. Wells which displayed potential suppression of toxin activity $(>39 \%)$ were verified by visual inspection to immediately exclude false hits arising from cellular toxicity, precipitation, or auto-fluorescence/quenching. Hits for confirmation and follow-up assays were ordered from Microsource and Sigma as lyophilized powders. Dose response curves were created and evaluated using Prism software (GraphPad Software, La Jolla, CA).

Acute toxicity assay (necrosis). Loss of cellular ATP as a marker of high dose $(1 \mathrm{nM}) \mathrm{TcdB}$ toxicity was measured as described for the Arrayscan screen protocol, except that CellTiter-Glo reagent (Promega, Madison, WI) was added to the cells 3-h post toxin challenge, and luminescence was recorded on a Spectramax M5 plate reader.

LysoTracker assay. Endosomal $\mathrm{pH}$ neutralization was assayed essentially as described by Slater et al. ${ }^{58}$; IMR90 cells in complete EMEM were plated at 14,000 cells/well ( $~ 95 \%$ confluency). After $24 \mathrm{~h}$, the media was changed to SFM for $60 \mathrm{~min}$, then compound was added to $40 \mu \mathrm{M}$ and incubated at $37^{\circ} \mathrm{C}$ for $2 \mathrm{~h}$. LysoTracker red DND-99 and Hoechst (Life Technologies) were added to 0.1 and $1 \mu \mathrm{M}$, respectively, and incubated for $60 \mathrm{~min}$. Excess dye was removed by media change and the fluorescence at ex/em 574/594 was read on an Envision plate reader (Perkin Elmer). Representative cell images were taken using a Zeiss Axiovert fluorescence microscope using DAPI and Texas Red filters to visualize the Hoechst and LysoTracker staining, respectively.

CDT toxicity assay. Vero cells were plated in 96-well Cellbind plates at a density of 8000 cells/well ( $\sim 90 \%$ confluent). The next day, compound dilutions and CDT binary toxins $(2 \mu \mathrm{g} / \mathrm{mL}$ each of $\mathrm{A}$ and trypsin-activated B-a kind gift from Merck) in serum-free DMEM were added to the plate. After $24 \mathrm{~h}$, wells were washed with PBS, and the cells were fixed with $4 \%$ paraformaldehyde for $15 \mathrm{~min}$, permeabilized with $0.25 \%$ TX100 for $5 \mathrm{~min}$, then F-actin was stained with Phalloidin Alexa488 (Thermo) for $2 \mathrm{~h}$ before washing and reading Alexa488 fluorescence on a Molecular Devices Spectramax M5e (bottom read, well scan, 9 points/well).

For photomicroscopy, the cells were stained with $1 \mu \mathrm{M}$ Hoechst for $30 \mathrm{~min}$, then combined photos were taken for each compound at a concentration corresponding to maximum protection from TcdB using a $10 \times$ objective and appropriate filter sets for Hoechst and Alexa488 fluorescence (Zeiss).

TcdB in vitro autoprocessing assay. Inhibition of TcdB self-cleavage by its intrinsic cysteine protease activity was measured by pre-incubating test compounds with TcdB for $30 \mathrm{~min}$, followed by addition of InsP6 and incubating the reaction at $37^{\circ} \mathrm{C}$ for $3 \mathrm{~h}$. Cleavage was visualized by electrophoresing the samples on SDS polyacrylamide gels and staining with Coomassie Blue R250.

Rac1 glucosylation. IMR90 cells were grown in 6-well plates at a density of 300,000 cells/well. The next day, TcdB ( $0.5 \mathrm{pM}$ final) and NEN (2A pharmachem) were added to the wells in serum-free media. After 60 min the cells were harvested and lysed in Laemmli buffer. SDS PAGE and western blotting were performed to detect glucosylated Rac1 using mAb102 (BD Biosciences), total Racl using mAb23A8 (Abcam), and loading controls using anti-EEA1 and anti-ERK1/2 (EMD Millipore). Raw Western Blot images are shown in Supplementary Figure 8.

Differential scanning fluorometry. Differential scanning fluorometry (DSF) was performed in a similar manner as described previously ${ }^{59}$. TcdB protein was diluted in phosphate buffer $\left(100 \mathrm{mM} \mathrm{KPO}_{4}, 150 \mathrm{mM} \mathrm{NaCl}, \mathrm{pH}\right.$ 7) containing $5 \times$ SYPRO Orange (Invitrogen, Burlington, $\mathrm{ON}$ ) and a serial dilution of test compound. A BioRad CFX96 qRT-PCR thermocycler was used to establish a temperature gradient from $15^{\circ} \mathrm{C}$ to $95^{\circ} \mathrm{C}$ in $30 \mathrm{~s}$ increments, while simultaneously recording the increase in SYPRO Orange fluorescence as a consequence of binding to hydrophobic regions exposed on unfolded proteins. The Bio-Rad CFX Manager 3.1 software was used to integrate the fluorescence curves to calculate the melting point.

TcdB cell surface binding. HCT116 cells in $10 \mathrm{~cm}$ dishes were grown to $90 \%$ confluence. TcdB $(2 \mathrm{nM})$ and either $100 \mu \mathrm{M}$ methylcholate or $1 \mu \mathrm{M}$ NEN were preincubated together for $30 \mathrm{~min}$ on ice in serum-free media before adding to cells After incubating for $60 \mathrm{~min}$ on ice, cells were washed with PBS, harvested and lysed in $300 \mu \mathrm{L}$ of $0.5 \%$ TX100/PBS. Clarified material was analyzed by western blot by probing with anti-TcdB antibody (R\&D Systems AF6246) and anti-tubulin antibody as a loading control. TcdB bands were measured by densitometry using a ChemiDoc MP Imaging System (BioRad).

UDP-Glo ${ }^{\mathrm{TM}}$ UDP-glucose hydrolase assay (Promega). Experiments were performed as per the manufacturer's instructions. Briefly, $100 \mathrm{nM}$ of GTD enzyme was incubated in glucosylation buffer (see above) with various concentrations of inhibitor in a final volume of $16 \mu \mathrm{L}$. Reactions were started with the addition of $4 \mu \mathrm{L}$ of UDP-glucose $(50 \mu \mathrm{M}$ final). Reactions were allowed to proceed at room 
temperature for $15 \mathrm{~min}$. To stop the reaction, $10 \mu \mathrm{L}$ were removed and added to a white, polystyrene 96-well half-area plate (Costar) containing $10 \mu \mathrm{L}$ of UDP detection reagent. Plates were incubated at room temperature for $1 \mathrm{~h}$, then luminescence was recorded on a SpectraMax M5e plate reader (Molecular Devices) with an integration time of $750 \mathrm{~ms}$. Results were analyzed with SoftMax Pro 6.2.2 and GraphPad Prism 5.0.

\section{MIC assays to determine effects of NEN on C. difficile strains. For results} shown in Table 2: Minimal Inhibitory Concentration (MIC) testing was performed by the National Research Council of Canada (Ottawa), using the following bacterial strains: Clostridium difficile R20291 (UK) O27 ribotype, Clostridium difficile M68 ${ }^{60}$ 017 ribotype, Clostridium clostridioforme ATCC25537, and Clostridium sporogenes ATCC3584. Compounds were dissolved in DMSO and added to Brucella supplemented blood plates. Niclosamide and NEN from a $10 \mathrm{mM}$ stock solution in DMSO, was tested at $50 \mu \mathrm{M}$ starting concentration (16 and $19 \mu \mathrm{g} / \mathrm{mL}$, respectively), with 1 in 3 serial dilutions. Vancomycin was tested as a positive control starting at $8 \mu \mathrm{g} / \mathrm{mL}$, with 1 in 2 serial dilutions.

Inoculum preparation: strains were grown on Brucella supplemented blood agar plates in an anaerobic chamber for $16 \mathrm{~h}$. For each strain, cells were harvested into saline to OD 0.1 . The $\mathrm{CFU} / \mathrm{mL}$ was confirmed by serial dilution and plating onto Brucella supplemented blood or Braziers agar for each experimental group.

For MIC Testing, $10 \mu \mathrm{L}$ of each strain was spotted three times onto each agar plate and incubated at $37^{\circ} \mathrm{C}$ in an anaerobic chamber. Each plate was examined for visible growth at $16,24,32$, and $48 \mathrm{~h}$. No further change in growth pattern was observed for any strain from 16 to $48 \mathrm{~h}$ growth. MIC determinations were performed in duplicate and reported as the concentration that completely inhibits growth.

For results represented in Table 3: Minimal Inhibitory Concentration (MIC) testing was performed by Micromyx. NEN and comparator antibiotics were prepared on the day of testing using solvents recommended by CLSI. Stock solutions of all compounds were made at $100 \times$ the final testing concentration. Test organisms consisted of clinical isolates from the American Type Culture Collection (ATCC) and Micromyx repository. Drug dilutions and drug-supplemented agar plates were prepared manually. After pouring the Supplemented Brucella agar plates, they were allowed to dry, pre-reduced in the Bactron II anaerobic chamber, then spot-inoculated using a Steers Replicator, yielding a final cell concentration on the surface of the agar plates of $\sim 1 \times 10^{4}$ colony-forming units/spot. After the inocula had dried, the drug-supplemented plates were incubated at $35^{\circ} \mathrm{C}$ for 16,24 , 32 , and $48 \mathrm{~h}$ under anaerobic conditions. The MIC was read per CLSI guidelines as the concentration at which growth was significantly inhibited relative to the growth control.

In vivo studies. All procedures involving animals were conducted under protocols approved by the Institutional Animal Care and Use Committee at the University of Maryland, Baltimore.

For the primary CDI Model ${ }^{61-64}, \mathrm{C} 57 \mathrm{BL} / 6$ mice (10 per group) were orally administered $10^{5} \mathrm{CFU}$ of $C$. difficile spores from the UK1 (BI/NAP1/027) strain after receiving antibiotic treatment in the drinking water for 3 days, as shown in Fig. 3. The antibiotic mixture contains: kanamycin $(0.4 \mathrm{mg}$ per $\mathrm{mL})$, gentamicin $(0.035 \mathrm{mg}$ per $\mathrm{mL})$, colistin $(850 \mathrm{U}$ per $\mathrm{mL})$, metronidazole $(0.215 \mathrm{mg}$ per $\mathrm{mL})$, and vancomycin $(0.045 \mathrm{mg}$ per $\mathrm{mL})$ was prepared. This corresponds to the approximate daily dose used for each antibiotic such as kanamycin (40 mg per $\mathrm{kg}$ ), gentamicin (3.5 mg per $\mathrm{kg}$ ), colistin (4.2 mg per $\mathrm{kg}$ ), metronidazole $(21.5 \mathrm{mg}$ per $\mathrm{kg}$ ), and vancomycin ( $4.5 \mathrm{mg}$ per $\mathrm{kg}$ ). Mice were given an intraperitoneal injection of clindamycin (10 mg per $\mathrm{kg}$ ) 1 day before spores challenge. Mouse weights and the development of disease symptoms were monitored daily. Animals that became moribund or lost $>20 \%$ of their body weight were euthanized. The mice were divided into the following groups: Control (water with 5\% DMSO), NEN $(2,10$, or $50 \mathrm{mg}$ per $\mathrm{kg}$ suspended in water with 5\% DMSO)

For the recurrent CDI Model, C57BL/6 mice (10 per group) were prepared for primary CDI model as previously mentioned; however, mice were orally administered vancomycin $(0.45 \mathrm{mg}$ per $\mathrm{mL})$ in the drinking water for 7 days post spores challenge. Mice were given regular water for until the end of the study. Mouse weights and the development of disease symptoms were monitored daily. Animals that became moribund or lost $>20 \%$ of their body weight were euthanized. Diarrhea was scored as following: 0 (normal hard fecal pellet); 1 (hard to produce fecal pellet yet no rectal inflammation); 2 (liquid feces, inflamed rectum, soiled tail).

Microbiota analysis. DNA was extracted using the MagAttract PowerMicrobiome DNA/RNA kit (Qiagen) from the fecal pellet of all samples. Briefly, the glass bead plate was used to mix fecal material and lysis solution, and inhibitor was subsequently removed from the supernatant. ClearMag Beads suspension was then mixed with $450 \mu \mathrm{L}$ of the supernatant to purify the extracted DNA.

DNA was extracted from all samples $(150 \mathrm{mg})$ using the MagAttract PowerMicrobiome DNA/RNA kit (Qiagen) implemented on a Hamilton STAR robotic platform and after a bead-beating step on a TissueLyzer II (Qiagen) in 96deep well plates. PCR amplification of the $16 \mathrm{~S}$ rRNA gene V4 hypervariable region was performed using dual-barcoded universal primers $515 \mathrm{~F}$

(GTGYCAGCMGCCGCGGTAA) and 806R (GGACTACNVGGGTWTCTAAT) as previously described ${ }^{65}$. High-throughput sequencing of the amplicons was performed on an Illumina MiSeq platform using the $300 \mathrm{bp}$ paired-end protocol. Raw data was demultiplexed using the idemp tool ${ }^{66}$. Barcode, adapter, and primer sequences were trimmed using tagcleaner ${ }^{67}$. Quality assessment and sequencing error correction was performed using the software package DADA2 ${ }^{68}$ and the following parameters: forward reads were truncated at position 220 and the reverse reads at position 160 based on the sequencing quality plot, no ambiguous based and a maximum of 2 expected errors per-read were allowed ${ }^{69}$. The qualitytrimmed reads were used to infer ribosomal sequence variants and their relative abundance in each sample after removing chimera. A total of 205 fecal samples were characterized resulting in a total of 6,619,465 high-quality non-chimeric amplicon sequences, corresponding to $32,290( \pm 19,999)$ sequences per samples. The GreenGene database version $13.8^{70}$ was used for taxonomic classification. Within-sample diversity was estimated using Shannon diversity index ${ }^{71}$. Intercommunity comparative analyses were performed using NMDS (nonmetric dimensional scaling) based on Bray-Curtis distance metrics and were plotted using software package phyloseq ${ }^{72}$. Linear discriminant analysis (LDA) effect size (LEfSe) analysis ${ }^{39}$ was applied to identify bacterial phylotypes with relative abundance statistically different between control and NEN (50 mg per $\mathrm{kg}$ ) treatments. The alpha value for the non-parametric factorial Kruskal-Wallis (KW) sum-rank test ${ }^{73}$ was 0.05 and the threshold for the logarithmic LDA model ${ }^{74}$ score for discriminative features was set at 2.0 .

\section{Data availability}

All 16S rRNA sequence data generated and analyzed during the current study were deposited and are available in SRA under BioProject PRJNA423011 (SRP128045).

Received: 9 July 2018 Accepted: 19 November 2018

Published online: 07 December 2018

\section{References}

1. Theriot, C. M., Bowman, A. A. \& Young, V. B. Antibiotic-induced alterations of the gut microbiota alter secondary bile acid production and allow for Clostridium difficile spore germination and outgrowth in the large intestine. mSphere 1, 00045-15 (2016).

2. McDonald, L. C. et al. An epidemic, toxin gene-variant strain of Clostridium difficile. N. Engl. J. Med. 353, 2433-2441 (2005)

3. Goorhuis, A. et al. Emergence of Clostridium difficile infection due to a new hypervirulent strain, polymerase chain reaction ribotype 078. Clin. Infect. Dis. 47, 1162-1170 (2008)

4. Gerding, D. N., Johnson, S., Rupnik, M. \& Aktories, K. Clostridium difficile binary toxin CDT: mechanism, epidemiology, and potential clinical importance. Gut Microbes 5, 15-27 (2014).

5. He, M. et al. Emergence and global spread of epidemic healthcare-associated Clostridium difficile. Nat. Genet. 45, 109-113 (2013).

6. Warny, M. et al. Toxin production by an emerging strain of Clostridium difficile associated with outbreaks of severe disease in North America and Europe. Lancet 366, 1079-1084 (2005).

7. Collins, J. et al. Dietary trehalose enhances virulence of epidemic Clostridium difficile. Nature 291-294 (2018).

8. Lanis, J. M., Barua, S. \& Ballard, J. D. Variations in TcdB activity and the hypervirulence of emerging strains of Clostridium difficile. PLoS Pathog. 6 e1001061 (2010).

9. Lyras, D. et al. Toxin B is essential for virulence of Clostridium difficile. Nature 458, 1176-1179 (2009).

10. Kuehne, S. A. et al. The role of toxin A and toxin B in Clostridium difficile infection. Nature 467, 711-713 (2010).

11. Kuehne, S. A. et al. Importance of toxin A, toxin B, and CDT in virulence of an epidemic Clostridium difficile strain. J. Infect. Dis. 209, 83-86 (2014).

12. Louie, T. M. et al. Clostridium difficile-associated diarrhea (CDAD). in Program and Abstracts of the 47th Interscience Conference on Antimicrobial Agents and Chemotherapy, poster K-425a, p. 212 (American Society for Microbiology, Washington, DC, 2007).

13. Zhang, Z. et al. Toxin-mediated paracellular transport of antitoxin antibodies facilitates protection against Clostridium difficile infection. Infect. Immun. $\mathbf{8 3}$ 405-416 (2015).

14. Kufel, W. D., Devanathan, A. S., Marx, A. H., Weber, D. J. \& Daniels, L. M. Bezlotoxumab: a novel agent for the prevention of recurrent Clostridium difficile infection. Pharmacotherapy 37, 1298-1308 (2017).

15. Wilcox, M. H. et al. Bezlotoxumab for prevention of recurrent Clostridium difficile infection. N. Engl. J. Med. 376, 305-317 (2017).

16. Spigaglia, P. et al. Clostridium difficile causing pediatric infections: new findings from a hospital-based study in Italy. Anaerobe 262-268 (2017)

17. Spigaglia, P., Barbanti, F., Morandi, M., Moro, M. L. \& Mastrantonio, P. Diagnostic testing for Clostridium difficile in Italian microbiological laboratories. Anaerobe 37, 29-33 (2016). 
18. Androga, G. O. et al. Infection with toxin A-negative, toxin B-negative, binary toxin-positive Clostridium difficile in a young patient with ulcerative colitis. J. Clin. Microbiol. 53, 3702-3704 (2015).

19. Grandesso, S. et al. Clostridium difficile ribotype 033 colitis in a patient following broad-spectrum antibiotic treatment for KPC producing Klebsiella pneumoniae infection, Italy. New Microbiol. 39, 235-236 (2016).

20. Eckert, C. et al. Prevalence and pathogenicity of binary toxin-positive Clostridium difficile strains that do not produce toxins A and B. New Microbes New Infect. 3, 12-17 (2015).

21. Chen, C. Z. et al. High-throughput Giardia lamblia viability assay using bioluminescent ATP content measurements. Antimicrob. Agents Chemother. 55, 667-675 (2011).

22. Xu, M. et al. Identification of small-molecule inhibitors of Zika virus infection and induced neural cell death via a drug repurposing screen. Nat. Med. 22, 1101-1107 (2016).

23. Johansen, L. M. et al. A screen of approved drugs and molecular probes identifies therapeutics with anti-Ebola virus activity. Sci. Transl. Med. 7, 290 ra289 (2015).

24. He, S. et al. Repurposing of the antihistamine chlorcyclizine and related compounds for treatment of hepatitis C virus infection. Sci. Transl. Med. 7, 282ra249 (2015).

25. Pearson, R. D. \& Hewlett, E. L. Niclosamide therapy for tapeworm infections. Ann. Intern. Med. 102, 550-551 (1985).

26. Andrews, P., Thyssen, J. \& Lorke, D. The biology and toxicology of molluscicides, Bayluscide. Pharmacol. Ther. 19, 245-295 (1982).

27. Hecht, G. \& Gloxhuber, C. [Studies on the tolerance of 5,2'-dichloro- $4^{\prime}$ nitrosalicylanilide ethanolamine salt]. Z. Trop. Parasitol. 13, 1-8 (1962).

28. Donta, S. T., Sullivan, N. \& Wilkins, T. D. Differential effects of Clostridium difficile toxins on tissue-cultured cells. J. Clin. Microbiol. 15, 1157-1158 (1982).

29. Qa'Dan, M. et al. Clostridium difficile toxin B activates dual caspase-dependent and caspase-independent apoptosis in intoxicated cells. Cell. Microbiol. 4, 425-434 (2002).

30. Tam, J. et al. Small molecule inhibitors of Clostridium difficile toxin B-induced cellular damage. Chem. Biol. 22, 175-185 (2015).

31. Eniu, A., Torode, J., Magrini, N. \& Bricalli, G., Union for International Cancer Control EML Steering Committee Members. Back to the 'essence' of medical treatment in oncology: the 2015 WHO Model List of Essential Medicines. ESMO Open 1, e000030 (2016).

32. Farrow, M. A. et al. Clostridium difficile toxin B-induced necrosis is mediated by the host epithelial cell NADPH oxidase complex. Proc. Natl. Acad. Sci. U.S. A. 110, 18674-18679 (2013).

33. Zhang, Z. et al. Translocation domain mutations affecting cellular toxicity identify the Clostridium difficile toxin B pore. Proc. Natl. Acad. Sci. U.S.A. 111, 3721-3726 (2014).

34. Jurgeit, A. et al. Niclosamide is a proton carrier and targets acidic endosomes with broad antiviral effects. PLoS Pathog. 8, e1002976 (2012).

35. Gooyit, M. \& Janda, K. D. Reprofiled anthelmintics abate hypervirulent stationary-phase Clostridium difficile. Sci. Rep. 6, 33642 (2016).

36. Pauk, K. et al. New derivatives of salicylamides: preparation and antimicrobial activity against various bacterial species. Bioorg. Med. Chem. 21, 6574-6581 (2013).

37. Hastey, C. J. et al. Comparison of Clostridium difficile minimum inhibitory concentrations obtained using agar dilution vs broth microdilution methods. Anaerobe 44, 73-77 (2017).

38. Tannock, G. W. et al. A new macrocyclic antibiotic, fidaxomicin (OPT-80), causes less alteration to the bowel microbiota of Clostridium difficile-infected patients than does vancomycin. Microbiology 156, 3354-3359 (2010).

39. Segata, N. et al. Metagenomic biomarker discovery and explanation. Genome Biol. 12, R60 (2011).

40. Slimings, C. \& Riley, T. V. Antibiotics and hospital-acquired Clostridium difficile infection: update of systematic review and meta-analysis. $J$. Antimicrob. Chemother. 69, 881-891 (2014).

41. Beilhartz, G. L., Tam, J. \& Melnyk, R. A. Small molecules take a big step against Clostridium difficile. Trends Microbiol. 23, 746-748 (2015).

42. Beilhartz, G. L., Tam, J., Zhang, Z. \& Melnyk, R. A. Comment on "A smallmolecule antivirulence agent for treating Clostridium difficile infection”. Sci. Transl. Med. 8, 370tc372 (2016).

43. Bender, K. O. et al. A small-molecule antivirulence agent for treating Clostridium difficile infection. Sci. Transl. Med. 7, 306ra148 (2015).

44. Carter, G. P. et al. Defining the roles of TcdA and TcdB in localized gastrointestinal disease, systemic organ damage, and the host response during Clostridium difficile infections. mBio 6, e00551 (2015).

45. Suliman, M. A. et al. Niclosamide inhibits colon cancer progression through downregulation of the Notch pathway and upregulation of the tumor suppressor miR-200 family. Int. J. Mol. Med. 38, 776-784 (2016)

46. Chen, L., Wang, L., Shen, H., Lin, H. \& Li, D. Anthelminthic drug niclosamide sensitizes the responsiveness of cervical cancer cells to paclitaxel via oxidative stress-mediated mTOR inhibition. Biochem. Biophys. Res. Commun. 484 , 416-421 (2017).

47. Xiang, M. et al. Niclosamide enhances the antitumor effects of radiation by inhibiting the hypoxia-inducible factor-1alpha/vascular endothelial growth factor signaling pathway in human lung cancer cells. Oncol. Lett. 14, 1933-1938 (2017).

48. $\mathrm{Yu}, \mathrm{X}$. et al. Niclosamide exhibits potent anticancer activity and synergizes with sorafenib in human renal cell cancer cells. Cell. Physiol. Biochem. 47, 957-971 (2018)

49. Zuo, Y. et al. Niclosamide enhances the cytotoxic effect of cisplatin in cisplatin-resistant human lung cancer cells via suppression of lung resistancerelated protein and c-myc. Mol. Med. Rep. 17, 3497-3502 (2018).

50. Tao, H., Zhang, Y., Zeng, X., Shulman, G. I. \& Jin, S. Niclosamide ethanolamine-induced mild mitochondrial uncoupling improves diabetic symptoms in mice. Nat. Med. 20, 1263-1269 (2014).

51. Lin, C. K. et al. Preclinical evaluation of a nanoformulated antihelminthic, niclosamide, in ovarian cancer. Oncotarget 7, 8993-9006 (2016).

52. Bhattacharyya, J. et al. Niclosamide-conjugated polypeptide nanoparticles inhibit Wnt signaling and colon cancer growth. Nanoscale 9, 12709-12717 (2017).

53. Naqvi, S., Mohiyuddin, S. \& Gopinath, P. Niclosamide loaded biodegradable chitosan nanocargoes: an in vitro study for potential application in cancer therapy. R. Soc. Open Sci. 4, 170611 (2017).

54. Brosschot, T. P. \& Reynolds, L. A. The impact of a helminth-modified microbiome on host immunity. Mucosal Immunol. 11, 1039-1046 (2018).

55. Reynolds, L. A. et al. Enteric helminths promote Salmonella coinfection by altering the intestinal metabolome. J. Infect. Dis. 215, 1245-1254 (2017).

56. Yang, G. et al. Expression of recombinant Clostridium difficile toxin A and B in Bacillus megaterium. BMC Microbiol. 8, 192 (2008).

57. Bagrodia, S., Taylor, S. J., Creasy, C. L., Chernoff, J. \& Cerione, R. A. Identification of a mouse p21Cdc42/Rac activated kinase. J. Biol. Chem. 270, 22731-22737 (1995).

58. Slater, L. H. et al. Identification of novel host-targeted compounds that protect from anthrax lethal toxin-induced cell death. ACS Chem. Biol. 8, 812-822 (2013).

59. Niesen, F. H., Berglund, H. \& Vedadi, M. The use of differential scanning fluorimetry to detect ligand interactions that promote protein stability. Nat. Protoc. 2, 2212-2221 (2007).

60. Ireland, P. M., Marshall, L., Norville, I. \& Sarkar-Tyson, M. The serine protease inhibitor Ecotin is required for full virulence of Burkholderia pseudomallei. Microb. Pathog. 67-68, 55-58 (2014).

61. Chen, X. et al. A mouse model of Clostridium difficile-associated disease Gastroenterology 135, 1984-1992 (2008).

62. Wang, $H$. et al. A chimeric toxin vaccine protects against primary and recurrent Clostridium difficile infection. Infect. Immun. 80, 2678-2688 (2012).

63. Yang, Z. et al. Mechanisms of protection against Clostridium difficile infection by the monoclonal antitoxin antibodies actoxumab and bezlotoxumab. Infect. Immun. 83, 822-831 (2015).

64. Yang, Z., Zhang, Y., Huang, T. \& Feng, H. Glucosyltransferase activity of Clostridium difficile Toxin B is essential for disease pathogenesis. Gut Microbes 6, 221-224 (2015)

65. Fadrosh, D. W. et al. An improved dual-indexing approach for multiplexed 16S rRNA gene sequencing on the Illumina MiSeq platform. Microbiome 2, 6 (2014).

66. Wu, Y. Barcode demultiplex for Illumina I1, R1, R2 fastq.gz files (https://github.com/yhwu/idemp). (2014).

67. Schmieder, R., Lim, Y. W., Rohwer, F. \& Edwards, R. TagCleaner: identification and removal of tag sequences from genomic and metagenomic datasets. BMC Bioinformatics 11, 341 (2010).

68. Callahan, B. J. et al. DADA2: high-resolution sample inference from Illumina amplicon data. Nat. Methods 13, 581-583 (2016).

69. Edgar, R. C. \& Flyvbjerg, H. Error filtering, pair assembly and error correction for next-generation sequencing reads. Bioinformatics 31, 3476-3482 (2015).

70. McDonald, D. et al. An improved Greengenes taxonomy with explicit ranks for ecological and evolutionary analyses of bacteria and archaea. ISME J. 6, 610-618 (2012).

71. Bent, S. J. \& Forney, L. J. The tragedy of the uncommon: understanding limitations in the analysis of microbial diversity. ISME J. 2, 689-695 (2008).

72. McMurdie, P. J. \& Holmes, S. phyloseq: an R package for reproducible interactive analysis and graphics of microbiome census data. PLoS One 8, e61217 (2013)

73. Kruskal, W. H. \& Wallis, W. A. Use of ranks in one-criterion variance analysis. J. Am. Stat. Assoc. 47, 583-621 (1952).

74. Fisher, R. A. The use of multiple measurements in taxonomic problems. Ann. Hum. Genet. 7, 179-188 (1936).

\section{Acknowledgements}

This work was supported by a Canadian Institutes of Health Research IndustryPartnered Collaborative Research Operating Grant (to R.A.M.). The authors thank 
Merck Research Laboratories for generous support in kind of reagents and materials. The authors also wish to thank Chris Fladd from the SPARC BioCentre at The Hospital for Sick Children for helpful assistance in setting up and developing the imaging-based screening platform, and Dr. Nachum Kaplan and Owen Roberts for their contributions towards studies related to evaluating the antimicrobial properties of niclosamide.

\section{Author contributions}

J.T., T.H., H.F., and R.A.M. designed the research. J.T., T.H., B.M., K.C., and G.L.B performed the research. J.T., T.H., B.M., K.C., G.L.B., J.R., H.F., and R.A.M analyzed the data and R.A.M. wrote the paper.

\section{Additional information}

Supplementary Information accompanies this paper at https://doi.org/10.1038/s41467018-07705-w.

Competing interests: The authors declare no competing interests.

Reprints and permission information is available online at http://npg.nature.com/ reprintsandpermissions/
Publisher's note: Springer Nature remains neutral with regard to jurisdictional claims in published maps and institutional affiliations.

(c) (i) Open Access This article is licensed under a Creative Commons Attribution 4.0 International License, which permits use, sharing, adaptation, distribution and reproduction in any medium or format, as long as you give appropriate credit to the original author(s) and the source, provide a link to the Creative Commons license, and indicate if changes were made. The images or other third party material in this article are included in the article's Creative Commons license, unless indicated otherwise in a credit line to the material. If material is not included in the article's Creative Commons license and your intended use is not permitted by statutory regulation or exceeds the permitted use, you will need to obtain permission directly from the copyright holder. To view a copy of this license, visit http://creativecommons.org/ licenses/by/4.0/.

(C) The Author(s) 2018 